\title{
Selective Formation of a Triangulo-Irondiplatinum Cluster
}

\author{
Cleber V. Ursini ${ }^{a}$, Gilson H.M. Dias ${ }^{a}$, Maria T.P. Gambardella ${ }^{b}$, \\ and Regina H. A. Santos \\ ${ }^{a}$ Instituto de Química, Universidade Estadual de Campinas, C.P. 6154, \\ 13083-970 Campinas - SP, Brazil \\ ${ }^{b}$ Instituto de Química de São Carlos, Universidade de São Paulo, C.P. 780, \\ 13560-250 São Carlos - SP, Brazil
}

Received: November 11, 1995

\begin{abstract}
O cluster de platina trinucleado $\left[\mathrm{Pt}_{3}\left(\mu-\mathrm{CNBu}^{\mathrm{t}}\right)_{3}\left(\mathrm{CNBu}^{\mathrm{t}}\right)_{3}\right]$ reage seletivamente com o complexo de ferro dppm-monodentado $[\mathrm{Fe}(\mathrm{dppm}-P)(\mathrm{CO}) 4]$, nas razões $1: 1$ ou $2: 1 \mathrm{de} \mathrm{Pt}: \mathrm{Fe}$ [dppm = bis(difenilfosfino)metano], para formar o cluster metálico misto [ $\mathrm{FePt}_{2}(\mu$-dppm $\left.)\left(\mathrm{CNBu}^{t}\right)_{2}(\mathrm{CO})_{4}\right]$. Um estudo de difração raios-X de um monocristal estabeleceu a sua estrutura como sendo um triângulo isósceles FePt2 com distâncias curtas Pt-Pt [2,5756(5) ̊̊], Fe-Pt(1) [2,559(2) ̊], e Fe-Pt(2) [2,565(3) $\AA$ ] , e com o ligante dppm ligado em ponte aos dois átomos de platina. Dados cristalográficos: sistema monoclínico; grupo espacial $P 21 / n ; a=11,031(1) \AA, b=20,593$ (3) $\AA$, $c=18,129(2)$ $\AA$. $\beta=91,32(1)^{\circ} ; D_{\mathrm{c}}(Z=4)=1,789 \mathrm{mg} \mathrm{m}^{-3}$. O refinamento por mínimos quadrados baseados em 4171 reflexões convergiu a $R=0,0508$ e $R_{\mathrm{W}}=0,0558$. Os espectros IV e RMN $\left({ }^{1} \mathrm{H},{ }^{13} \mathrm{C}-\left\{{ }^{1} \mathrm{H}\right\}\right.$, ${ }^{31} \mathrm{P}-\left\{{ }^{1} \mathrm{H}\right\}$, e $\left.{ }^{195} \mathrm{Pt}-\left\{{ }^{1} \mathrm{H}\right\}\right)$ do cluster são também fornecidos e discutidos.
\end{abstract}

The trinuclear platinum cluster $\left[\mathrm{Pt}_{3}\left(\mu-\mathrm{CNBu}^{\mathrm{t}}\right)_{3}\left(\mathrm{CNBu}^{\mathrm{t}}\right)_{3}\right]$ reacts selectively with the monodentate dppm-iron complex $[\mathrm{Fe}(\mathrm{dppm}-P)(\mathrm{CO}) 4](1: 1$ or $2: 1$ ratios of $\mathrm{Pt}: \mathrm{Fe})[\mathrm{dppm}=$ bis(diphenylphosphino)methane], to give the mixed-metal cluster $\left[\mathrm{FePt}_{2}(\mu-\mathrm{dppm})\left(\mathrm{CNBu}^{\mathrm{t}}\right)_{2}(\mathrm{CO})_{4}\right]$. A single-crystal X-ray diffraction study established the structure of an isosceles triangular $\mathrm{FePt}_{2}$ core

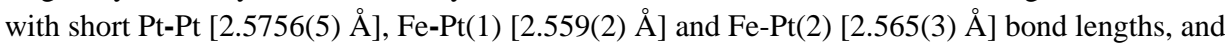
with the two platinum atoms bridged by the dppm ligand. Cell data: monoclinic, $P 21 / n, a=11.031$ (1) $\AA, b=20.593(3) \AA, c=18.129(2) \AA, \beta=91.32(1)^{\circ}, D_{\mathrm{c}}(Z=4)=1.789 \mathrm{mg} \mathrm{m}^{-3}$. Least-squares refinement based on 4171 reflections converged to a final $R=0.0508$ and $R_{\mathrm{W}}=0.0558$. The IR and NMR spectra $\left({ }^{1} \mathrm{H},{ }^{13} \mathrm{C}-\left\{{ }^{1} \mathrm{H}\right\},{ }^{31} \mathrm{P}-\left\{{ }^{1} \mathrm{H}\right\}\right.$, and $\left.{ }^{195} \mathrm{Pt}-\left\{{ }^{1} \mathrm{H}\right\}\right)$ of the cluster are also reported and discussed.

Keywords: trinuclear irondiplatinum cluster, ligand transfer

\section{Introduction}

Recent studies have attracted considerable interest in developing a rational stepwise assembly of organometallic building blocks and in understanding the factors that govern the selectivity of reactions ${ }^{1}$. In contrast with monophosphines that do not tend to prevent cluster fragmentation ${ }^{2}$, dppm as an assembling ligand greatly contributes to the stability of the resulting cluster molecule ${ }^{2 \mathrm{a}}$. The strong propensity of the dppm ligand for bridging between two metal centers has been useful to prepare mixed-metal cluster complexes with a five-membered M( $\mu$-dppm)M' unit ${ }^{3}$. The regioselective insertions of a metal carbonyl fragment towards bridging $[\operatorname{PtPd}(\mu-$ $\left.\mathrm{dppm})_{2} \mathrm{Cl}_{2}\right]$ and the four-membered chelating $[\mathrm{Pt}(\mathrm{dppm}-$ $\left.P P^{\prime}\right) \mathrm{Cl}_{2}$ ] compounds take place at the labile Pd-P and Pt-P bonds ${ }^{4,5}$.

A good iron precursor of heterobimetallic $\mathrm{Fe}(\mu$ dppm)Pt units is $\left[\mathrm{Fe}(\mathrm{dppm}-P)(\mathrm{CO})_{4}\right]$. Although it has been described as inert toward substitution by several organic substrates, the attack of the uncoordinated phosphorus on 
labile metallic centers occurs without the need of breaking the Fe-P bond ${ }^{6}$.

The results of our investigation of a selective reaction of the monodentate dppm-iron complex $[\mathrm{Fe}(\mathrm{dppm}-$ $\left.P)(\mathrm{CO})_{4}\right]$ with $\left[\mathrm{Pt}_{3}\left(\mu-\mathrm{CNBu}^{\mathrm{t}}\right)_{3}\left(\mathrm{CNBu}^{\mathrm{t}}\right)_{3}\right]$ to form the $\left[\mathrm{FePt}_{2}(\mu\right.$-dppm $\left.)\left(\mathrm{CNBu}^{\mathrm{t}}\right)_{2}(\mathrm{CO})_{4}\right]$ heterometallic cluster are described. However, in contrast to the usual reactions of the dppm-assisted metal-metal bond formation, a complete transfer of the dppm ligand from the iron precursor to the two Pt atoms is observed in this case.

\section{Results and Discussion}

\section{Synthesis and Characterization of $\left[\mathrm{FePt}_{2}(\mu-d p p m)\right.$ $\left.\left.(\mathrm{CNBu})^{\mathrm{t}}\right)_{2}(\mathrm{CO})_{4}\right]$}

The reactions of $\left[\mathrm{Pt}_{3}\left(\mu-\mathrm{CNBu}^{\mathrm{t}}\right)_{3}\left(\mathrm{CNBu}^{\mathrm{t}}\right)_{3}\right]$ with $\left[\mathrm{Fe}(\mathrm{dppm}-\mathrm{P})(\mathrm{CO})_{4}\right]$ (ratios $\mathrm{Fe}: \mathrm{Pt}$ of $1: 1$ or $1: 2$ ) in THF result initially in a deep dark green solution. After a period of $1.5 \mathrm{~h}$ at room temperature, analytically pure orange samples of $\left[\mathrm{FePt}_{2}(\mu\right.$-dppm $\left.)\left(\mathrm{CNBu}^{\mathrm{t}}\right)_{2}(\mathrm{CO})_{4}\right]$ are isolated in the $60-70 \%$ range from the orange reaction mixture. Spectroscopic data were consistent with the structure drawn for $\left[\mathrm{FePt}_{2}(\mu\right.$-dppm $\left.)\left(\mathrm{CNBu}^{\mathrm{t}}\right)_{2}(\mathrm{CO})_{4}\right]$, which was further confirmed by an X-ray diffraction study. The IR spectrum of $\left[\mathrm{FePt}_{2}(\mu\right.$-dppm $\left.)\left(\mathrm{CNBu}^{\mathrm{t}}\right)_{2}(\mathrm{CO})_{4}\right]$ displays $v(\mathrm{CO})$ absorptions of terminal and semi-bridged CO ligands at 1976, 1909 , and $1851 \mathrm{~cm}^{-1}$.

The ${ }^{195} \mathrm{Pt}(\mathrm{I}=1 / 2)$ isotope has a natural abundance of $33.8 \%$, corresponding to three isotopic distributions in a binuclear $\mathrm{Pt}_{2}(\mu$-dppm) arrangement: the zero-spin Pt isotopomer (43.95\%), mono (44.68\%) and doubly (11.35\%) labelled ${ }^{195} \mathrm{Pt}$ isotopomers ${ }^{7}$.

(A)

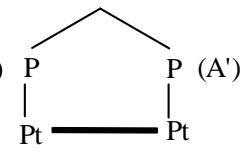

$43.95 \%$

$$
* \mathrm{Pt}={ }^{195} \mathrm{Pt}
$$

(A)

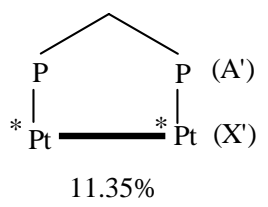

Therefore, a 1:7.8:17.4:7.8:1 quintet of triplets is expected for the $\mathrm{CH}_{2} \mathrm{P}$ group of the dppm ligand bonded to two $\mathrm{Pt}$ atoms in ${ }^{1} \mathrm{H}-\mathrm{NMR}$ spectrum. The observed spectrum was in good agreement with this prediction, although the weakest outer triplets with unit relative intensity were obscured by the noise.
The ${ }^{31} \mathrm{P}\left\{{ }^{1} \mathrm{H}\right\}$ NMR spectrum of $\left[\mathrm{FePt}_{2}(\mu\right.$-dppm) $\left.\left(\mathrm{CNBu}^{\mathrm{t}}\right)_{2}(\mathrm{CO})_{4}\right]$ displays a sharp singlet at $\delta-4.18$ with platinum satellites $\left[{ }^{1} J(\mathrm{PtP}) 3671 \mathrm{~Hz}\right]$, which confirms a symmetrical $\boldsymbol{A}$-frame $\mathrm{Pt}\left(\boldsymbol{\mu}\right.$-dppm)Pt moiety ${ }^{8}$. Moreover, the first indication of the presence of a strong Pt-Pt bond is provided by a large negative value $(75 \mathrm{~Hz})$ for ${ }^{2} J(\mathrm{PtP})$, showing through-bond contributions of ${ }^{2} J(\mathrm{PPtPt})$ to be dominant ${ }^{9}$.

The color change of the THF solution containing $\left[\mathrm{Pt}_{3}\left(\mu-\mathrm{CNBu}^{\mathrm{t}}\right)_{3}\left(\mathrm{CNBu}^{\mathrm{t}}\right)_{3}\right]$ and $\left[\mathrm{Fe}(\mathrm{dppm}-\mathrm{P})(\mathrm{CO})_{4}\right]$ from orange to green only occurs above $-22{ }^{\circ} \mathrm{C}$. The analytical data of the dark green solid produced in ethyl ether/petroleum ether at $-5{ }^{\circ} \mathrm{C}$, coincidentally pointed to $\left[\mathrm{FePt}_{2}(\mathrm{dppm})\left(\mathrm{CNBu}^{\mathrm{t}}\right)_{2}(\mathrm{CO})_{4}\right]$ formulation as the main cluster. However, the time ${ }^{31} \mathrm{P}-\left\{{ }^{1} \mathrm{H}\right\}$ NMR spectra exhibit the characteristic pattern of a $\mathrm{Pt}(\mu-\mathrm{dppm}) \mathrm{Fe}$ core $[\delta-10.5$ $(P P t)$ and $46.0(P f e)]$, that quickly converts towards $\left[\mathrm{FePt}_{2}(\mu\right.$-dppm $\left.)\left(\mathrm{CNBu}^{\mathrm{t}}\right)_{2}(\mathrm{CO})_{4}\right]$. On the basis of these spectroscopic and analytical data, therefore, the green intermediate can be analogous to the main cluster but with a $\operatorname{Pt}(\mu$-dppm)Fe unit.

Curiously, the reaction that yelds $\left[\mathrm{FePt}_{2}(\mu\right.$-dppm) $\left.\left(\mathrm{CNBu}^{\mathrm{t}}\right)_{2}(\mathrm{CO})_{4}\right]$ does not depend on the stoichiometry of the reagents. Even when the precursor $\left[\mathrm{Fe}(\mathrm{dppm}-\mathrm{P})(\mathrm{CO})_{4}\right]$ is used in excess, no by-product is further isolated. This contrasts, therefore, with the coupling reactions of $\left[\mathrm{Fe}(\mathrm{dppm}-\mathrm{P})(\mathrm{CO})_{4}\right]$ with $\left[\mathrm{Pt}\left(\mathrm{PR}_{3}\right)_{2}\left(\mathrm{C}_{2} \mathrm{H}_{4}\right)\right]$ for which dimeric complexes of the $\left[\mathrm{FePt}(\mu-\mathrm{CO})(\mu\right.$-dppm $)(\mathrm{CO})_{3}$ $\left.\left(\mathrm{PR}_{3}\right)\right]^{6}$ type form selectively. It is likely that the reactions take place via the primary dimeric intermediate $[\mathrm{FePt}(\mu$ $\mathrm{CO})(\mu$-dppm $\left.)(\mathrm{CO})_{3}\left(\mathrm{CNBu}^{t}\right)\right]$ with a $\mathrm{Fe}(\mu$-dppm)Pt moiety, although this was not spectroscopically observed. However, the iron-phosphorus bond breaking and phosphorus migration to the second platinum atom appears to occur after the formation of the green triangle-intermediate with a Fe( $\mu$-dppm)Pt unit, as illustrated in Scheme 1.

\section{$X$-Ray Diffraction study of $\left[\mathrm{FePt}_{2}(\mu-d p p m)\left(C N B u^{\mathrm{t}}\right)_{2}\right.$ $\left.(\mathrm{CO})_{4}\right]$}

An $O R T E P^{10}$ drawing of the molecular structure is shown in Fig. 1 with its atom numbering scheme. Selected bond distances and angles are given in Table 1. Final positional parameters are listed in Table 3.

The $\left[\mathrm{FePt}_{2}(\mu\right.$-dppm $\left.)\left(\mathrm{CNBu}^{t}\right)_{2}(\mathrm{CO})_{4}\right]$ complex contains an isosceles triangle of $\mathrm{FePt}_{2}$ atoms (mean $60.0^{\circ}$ ) with a dppm ligand bridging two platinum edges to form a roughly planar FePt 2 core. The $\mathrm{C}(\mathrm{A} 1) \mathrm{O}(\mathrm{A} 1)$ and $\mathrm{C}(\mathrm{A} 2) \mathrm{O}(\mathrm{A} 2)$ carbonyls, the isocyanide ligands, and phosphorus atoms of the dppm ligand are approximately coplanar with the metal triangle, but no longer collinear with the metal-metal axis. The bond angles between the $\mathrm{Fe}(\mathrm{C})_{2}$ and $\mathrm{Pt}(\mathrm{C})(\mathrm{P})$ planes [C(A1)-Fe-C(A2)], P(1)-Pt(1)-C(1) and P(2)-Pt(2)-C(2)] 


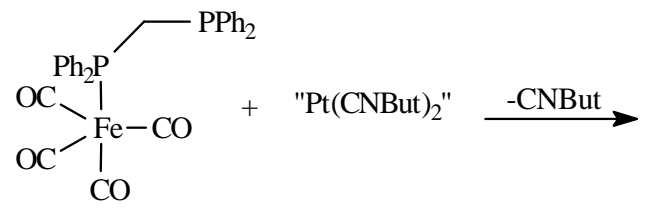

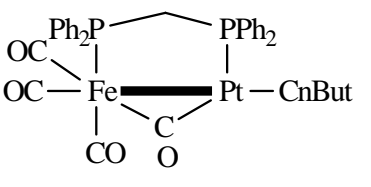

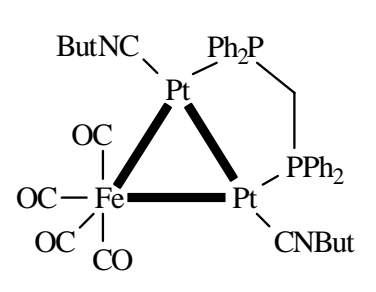

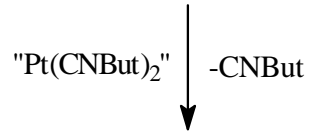

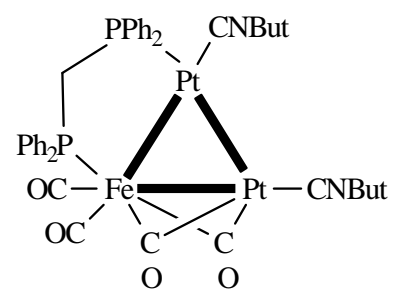

Scheme 1.

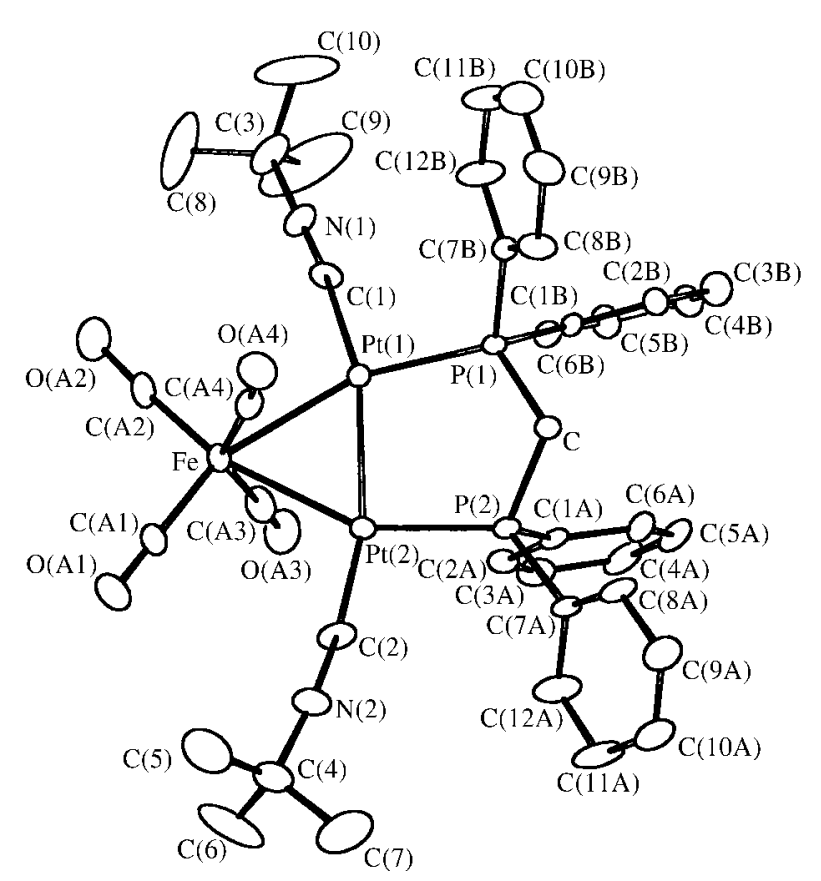

Figure 1. Molecular structure and atom labeling scheme for $\left[\mathrm{FePt}_{2}(\mu-\right.$ dppm $\left.)\left(\mathrm{CNBu}^{\mathrm{t}}\right)_{2}(\mathrm{CO}) 4\right]$. Thermal ellipsoids are shown at the $50 \%$ probability level.

have an average value of $102^{\circ}$. Another feature of note in the $\left[\mathrm{FePt}_{2}(\mu-\mathrm{dppm})\left(\mathrm{CNBu}^{\mathrm{t}}\right)_{2}(\mathrm{CO})_{4}\right]$ molecular structure and one that may influence the iron coordination geometry is the angular deformation of both apical carbonyl ligands bound to the $\mathrm{Fe}$ atom [C(A3)-Fe-C(A4) angle $143^{\circ}$ ], which are semi-bridging ${ }^{11}$, but not symmetrically arranged

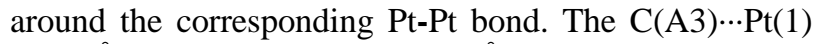
$(2.51 \AA)$ and $\mathrm{C}(\mathrm{A} 4) \cdots \mathrm{Pt}(2)(2.68 \AA)$ distances are shorter

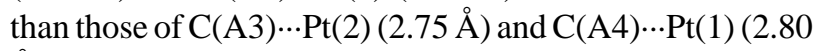
$\AA)$, respectively. Therefore, the geometry adopted by the iron in the $\mathrm{Pt}_{2} \mathrm{Fe}(\mathrm{CO})_{4}$ moiety deviates slightly from octahedral symmetry. The distortion is toward a bicapped tet- rahedral disposition of the four carbonyl groups, with the platinum atoms capping two tetrahedral faces. The Pt-Pt [2.5756(5) $\AA]$ and Fe-Pt (mean 2.563(2) $\AA$ ) bonds lengths found in $\left[\mathrm{FePt}_{2}(\mu\right.$-dppm $\left.)\left(\mathrm{CNBu}^{t}\right)_{2}(\mathrm{CO})_{4}\right]$ lie around the lower limit range reported for formal single bonds in other mixed platinum-iron compounds (Pt-Pt 2.565 $\AA$ and Pt-Fe $2.530 \AA)^{1 \mathrm{c}, 5 \mathrm{c}, 12}$. The contraction of metal-metal separations by bridging $\mathrm{CO}$ and CNR groups is commonly observed $^{1 \mathrm{~b}, 13}$ and may attribute in part to the small radius of the bridging carbon atoms since those distances are found to depend mostly on the size of the atoms of the bridging ligands ${ }^{11 \mathrm{~d}}$. All the internal P-C-P $\left[106.6(5)^{\circ}\right]$ and Pt-Pt-P angles $\left[95.83(7)^{\circ}\right.$ and $\left.90.96(7)^{\circ}\right]$ are close to those reported in unidentate dppm complexes ${ }^{14}$, indicating no particular strain of the five membered ring besides the usual clashing between phenyl groups in axial sites on the envelope $\mathrm{Pt}_{2} \mathrm{P}_{2} \mathrm{C}$ ring ${ }^{15}$. The terminal isocyanide groups are effectively linear [C-N-C and Pt-C-N $\left(\right.$ mean $\left.\left.175^{\circ}\right)\right]$. The average Fe-C and C-O distances are $1.76 \AA$ and $1.18 \AA$, respectively. The other bond angles and distances are as expected (Table 1). The architecture of $\left[\mathrm{FePt}_{2}(\mu-\mathrm{dppm})\left(\mathrm{CNBu}^{t}\right)_{2}\right.$ $\left.(\mathrm{CO})_{4}\right]$ bears a strong resemblance to that found for the $\left[\mathrm{FePt}_{2}(\mu-\mathrm{dppm})(\mathrm{CO})_{6}\right]^{5 \mathrm{c}}$ cluster.

\section{Conclusion}

This work shows the assisting role of the dppm ligand in building triangular $\left[\mathrm{FePt}_{2}(\mu\right.$-dppm $\left.)\left(\mathrm{CNBu}^{t}\right)_{2}(\mathrm{CO})_{4}\right]$ cluster when $\left[\mathrm{Fe}(\mathrm{dppm}-P)(\mathrm{CO})_{4}\right]$ reacts with $\left[\mathrm{Pt}_{3}(\mu-\right.$ $\left.\left.\mathrm{CNBu}^{t}\right)_{3}\left(\mathrm{CNBu}^{\mathrm{t}}\right)_{3}\right]$.

\section{Experimental}

\section{General considerations}

All manipulations were performed using general Schlenk and highvacuum techniques under a dry, oxygenfree, argon atmosphere. Solvents were appropriately dried and distilled under argon prior to use. The $\left[\mathrm{Pt}(\mathrm{COD})_{2}\right]^{16}$ 
Table 1. Selected Bond Distances $(\AA)$ and Angles $(\mathrm{deg})$ for $\left[\mathrm{FePt}_{2}(\mu-\mathrm{dppm})\left(\mathrm{CNBu}^{\mathrm{t}}\right)_{2}(\mathrm{CO}) 4\right]$ with Esd's in parentheses.

\begin{tabular}{|c|c|c|c|}
\hline $\operatorname{Pt}(1)-\operatorname{Pt}(2)$ & $2.5756(5)$ & & \\
\hline $\mathrm{Pt}(1)-\mathrm{Fe}$ & $2.559(2)$ & $\mathrm{Pt}(2)-\mathrm{Fe}$ & $2.565(2)$ \\
\hline $\mathrm{Pt}(1)-\mathrm{P}(1)$ & $2.233(2)$ & $\mathrm{Pt}(2)-\mathrm{P}(2)$ & $2.235(3)$ \\
\hline $\operatorname{Pt}(1)-\mathrm{C}(1)$ & $1.95(2)$ & $\operatorname{Pt}(2)-C(2)$ & $1.92(2)$ \\
\hline $\mathrm{Fe}-\mathrm{C}(\mathrm{A} 1)$ & $1.76(1)$ & $\mathrm{Fe}-\mathrm{C}(\mathrm{A} 2)$ & $1.73(1)$ \\
\hline $\mathrm{Fe}-\mathrm{C}(\mathrm{A} 3)$ & $1.77(1)$ & $\mathrm{Fe}-\mathrm{C}(\mathrm{A} 4)$ & $1.77(1)$ \\
\hline $\mathrm{P}(1)-\mathrm{C}$ & $1.847(9)$ & $\mathrm{P}(2)-\mathrm{C}$ & $1.80(1)$ \\
\hline $\mathrm{P}(1)-\mathrm{C}(1 \mathrm{~B})$ & $1.82(1)$ & $\mathrm{P}(2)-\mathrm{C}(1 \mathrm{~A})$ & $1.82(2)$ \\
\hline $\mathrm{P}(1)-\mathrm{C}(7 \mathrm{~B})$ & $1.84(9)$ & $\mathrm{P}(2)-\mathrm{C}(7 \mathrm{~A})$ & $1.84(2)$ \\
\hline $\mathrm{C}(\mathrm{A} 1)-\mathrm{O}(\mathrm{A} 1)$ & $1.16(2)$ & $\mathrm{C}(\mathrm{A} 2)-\mathrm{O}(\mathrm{A} 2)$ & $1.20(2)$ \\
\hline $\mathrm{C}(\mathrm{A} 3)-\mathrm{O}(\mathrm{A} 3)$ & $1.17(2)$ & $\mathrm{C}(\mathrm{A} 4)-\mathrm{O}(\mathrm{A} 4)$ & $1.17(2)$ \\
\hline $\mathrm{C}(1)-\mathrm{N}(1)$ & $1.16(1)$ & $\mathrm{C}(2)-\mathrm{N}(2)$ & $1.18(2)$ \\
\hline $\mathrm{N}(1)-\mathrm{C}(3)$ & $1.44(2)$ & $\mathrm{N}(2)-\mathrm{C}(4)$ & $1.47(2)$ \\
\hline $\mathrm{Pt}(1)-\mathrm{Fe}-\mathrm{Pt}(2)$ & $60.35(4)$ & & \\
\hline $\mathrm{Pt}(1)-\mathrm{Fe}-\mathrm{C}(\mathrm{A} 2)$ & $102.1(4)$ & $\mathrm{Pt}(2)-\mathrm{Fe}-\mathrm{C}(\mathrm{A} 2)$ & $161.6(4)$ \\
\hline $\mathrm{Pt}(1)-\mathrm{Fe}-\mathrm{C}(\mathrm{A} 3)$ & $68.3(5)$ & $\mathrm{Pt}(2)-\mathrm{Fe}-\mathrm{C}(\mathrm{A} 3)$ & $76.3(4)$ \\
\hline $\mathrm{Pt}(1)-\mathrm{Fe}-\mathrm{C}(\mathrm{A} 1)$ & $154.9(4)$ & $\mathrm{Pt}(2)-\mathrm{Fe}-\mathrm{C}(\mathrm{A} 1)$ & $95.6(4)$ \\
\hline $\mathrm{Pt}(1)-\mathrm{Fe}-\mathrm{C}(\mathrm{A} 4)$ & $78.3(4)$ & $\mathrm{Pt}(2)-\mathrm{Fe}-\mathrm{C}(\mathrm{A} 4)$ & $73.9(4)$ \\
\hline $\mathrm{C}(\mathrm{A} 1)-\mathrm{Fe}-\mathrm{C}(\mathrm{A} 2)$ & $102.5(6)$ & $\mathrm{C}(\mathrm{A} 2)-\mathrm{Fe}-\mathrm{C}(\mathrm{A} 3)$ & $103.1(6)$ \\
\hline $\mathrm{C}(\mathrm{A} 1)-\mathrm{Fe}-\mathrm{C}(\mathrm{A} 3)$ & $100.9(5)$ & $\mathrm{C}(\mathrm{A} 2)-\mathrm{Fe}-\mathrm{C}(\mathrm{A} 4)$ & $98.6(7)$ \\
\hline $\mathrm{C}(\mathrm{A} 1)-\mathrm{Fe}-\mathrm{C}(\mathrm{A} 4)$ & $103.2(6)$ & $\mathrm{C}(\mathrm{A} 3)-\mathrm{Fe}-\mathrm{C}(\mathrm{A} 4)$ & $143.1(5)$ \\
\hline $\mathrm{Fe}-\mathrm{C}(\mathrm{A} 1)-\mathrm{O}(\mathrm{A} 1)$ & $177(1)$ & $\mathrm{Fe}-\mathrm{C}(\mathrm{A} 1)-\mathrm{O}(\mathrm{A} 1)$ & $170(1)$ \\
\hline $\mathrm{Fe}-\mathrm{C}(\mathrm{A} 2)-\mathrm{O}(\mathrm{A} 2)$ & $175(1)$ & $\mathrm{Fe}-\mathrm{C}(\mathrm{A} 2)-\mathrm{O}(\mathrm{A} 2)$ & $175(2)$ \\
\hline $\operatorname{Pt}(2)-\operatorname{Pt}(1)-\mathrm{P}(1)$ & $95.83(7)$ & $\mathrm{Pt}(1)-\mathrm{Pt}(2)-\mathrm{P}(2)$ & $90.96(7)$ \\
\hline $\operatorname{Pt}(2)-\operatorname{Pt}(1)-C(1)$ & $163.1(4)$ & $\mathrm{Pt}(1)-\mathrm{Pt}(2)-\mathrm{C}(2)$ & $166.6(4)$ \\
\hline $\mathrm{Pt}(2)-\mathrm{Pt}(1)-\mathrm{Fe}$ & $59.94(5)$ & $\mathrm{Pt}(1)-\mathrm{Pt}(2)-\mathrm{Fe}$ & $59.71(5)$ \\
\hline $\mathrm{Fe}-\mathrm{Pt}(1)-\mathrm{P}(1)$ & $152.75(8)$ & $\mathrm{Fe}-\mathrm{Pt}(2)-\mathrm{P}(2)$ & $150.30(8)$ \\
\hline $\mathrm{Fe}-\mathrm{Pt}(1)-\mathrm{C}(1)$ & $105.2(4)$ & $\mathrm{Fe}(1)-\operatorname{Pt}(2)-\mathrm{C}(2)$ & $107.1(4)$ \\
\hline $\mathrm{P}(1)-\mathrm{Pt}(1)-\mathrm{C}(1)$ & $100.3(4)$ & $\mathrm{P}(2)-\mathrm{Pt}(2)-\mathrm{C}(2)$ & $102.4(4)$ \\
\hline $\mathrm{Pt}(1)-\mathrm{P}(1)-\mathrm{C}$ & $112.8(4)$ & $\mathrm{Pt}(2)-\mathrm{P}(2)-\mathrm{C}$ & $110.9(3)$ \\
\hline $\mathrm{Pt}(1)-\mathrm{P}(1)-\mathrm{C}(1 \mathrm{~B})$ & $119.2(4)$ & $\mathrm{Pt}(2)-\mathrm{P}(2)-\mathrm{C}(1 \mathrm{~A})$ & $113.9(3)$ \\
\hline $\mathrm{Pt}(1)-\mathrm{P}(1)-\mathrm{C}(7 \mathrm{~B})$ & $114.3(3)$ & $\mathrm{Pt}(2)-\mathrm{P}(2)-\mathrm{C}(7 \mathrm{~A})$ & $120.3(3)$ \\
\hline $\mathrm{C}-\mathrm{P}(1)-\mathrm{C}(1 \mathrm{~B})$ & $103.0(4)$ & $\mathrm{C}-\mathrm{P}(2)-\mathrm{C}(1 \mathrm{~A})$ & $104.1(5)$ \\
\hline C-P(1)-C(7B) & $104.6(5)$ & $\mathrm{C}-\mathrm{P}(2)-\mathrm{C}(7 \mathrm{~A})$ & $104.6(5)$ \\
\hline $\mathrm{C}(1 \mathrm{~B})-\mathrm{P}(1)-(7 \mathrm{~B})$ & $101.2(5)$ & $\mathrm{C}(1 \mathrm{~A})-\mathrm{P}(2)-\mathrm{C}(7 \mathrm{~A})$ & $101.4(5)$ \\
\hline $\mathrm{P}(1)-\mathrm{C}-\mathrm{P}(2)$ & $106.6(5)$ & & \\
\hline $\mathrm{Pt}(1)-\mathrm{C}(1)-\mathrm{N}(1)$ & $173(2)$ & $\mathrm{Pt}(2)-\mathrm{C}(2)-\mathrm{N}(2)$ & $173(1)$ \\
\hline $\mathrm{C}(1)-\mathrm{N}(1)-\mathrm{C}(3)$ & $176(1)$ & $\mathrm{C}(2)-\mathrm{N}(2)-\mathrm{C}(4)$ & $173(1)$ \\
\hline
\end{tabular}

$(\mathrm{COD}=1,5$-cyclooctadiene $),\left[\mathrm{Pt}_{3}\left(\mu-\mathrm{CNBu}^{\mathrm{t}}\right)_{3}\left(\mathrm{CNBu}^{\mathrm{t}}\right)_{3}\right]^{17}$ and $\left[\mathrm{Fe}(\mathrm{CO})_{4}(\operatorname{dppm} P)\right]^{18}$ complexes were prepared as described previously. Other reagents were used as obtained from commercial sources. Elemental analyses were performed using a PERKINELMER $2400 \mathrm{CHN}$ microana- lyser. All percentages given are the average of at least two independent determinations. IR spectra were recorded as Nujol mulls with a JASCO IR 700 spectrometer. NMR spectra were obtained with a BRUKER WH 400 $\left({ }^{13} \mathrm{C}, 100.589 ;{ }^{31} \mathrm{P}, 161.796\right.$; and $\left.{ }^{195} \mathrm{Pt}, 85.629 \mathrm{MHz}\right)$ and a 
BRUKER AW $80\left({ }^{1} \mathrm{H}, 80 \mathrm{MHz}\right)$ instruments. The chemical shifts of ${ }^{1} \mathrm{H}$ and ${ }^{13} \mathrm{C}$ spectra were referenced to TMS, and the ${ }^{31} \mathrm{P}$ spectra were quoted relative to external $85 \% \mathrm{H}_{3} \mathrm{PO}_{4}$ at $0.00 \mathrm{ppm}$. Platinum-195 chemical shifts were quoted relative to the absolute scale $\Xi\left({ }^{195} \mathrm{Pt}\right)=21.4 \mathrm{MHz}$.

\section{Preparations of $\left[\mathrm{FePt}_{2}(\mu-\mathrm{dppm})\left(\mathrm{CNBu}^{\mathrm{t}}\right)_{2}(\mathrm{CO})_{4}\right]$}

The $\left[\mathrm{Pt}_{3}\left(\mu-\mathrm{CNBu}^{\mathrm{t}}\right)_{3}\left(\mathrm{CNBu}^{\mathrm{t}}\right)_{3}\right]$ complex $(0.162 \mathrm{~g}, 0.150$ mmol) was slowly added to a stirred solution of $\left[\mathrm{Fe}(\mathrm{CO})_{4}(\mathrm{dppm} P)\right](0.247 \mathrm{~g}, 0.447 \mathrm{mmol})$ in THF $(15 \mathrm{~mL})$ at $5{ }^{\circ} \mathrm{C}$. The mixture was allowed to reach room temperature over $1.5 \mathrm{~h}$. The color gradually changed from deep black to orange. Filtration of the solution with a short alumina column, and evaporation to $3 \mathrm{~mL}$ followed by addition of petroleum ether resulted in orange microcrystals. The product was washed with petroleum ether $(3 \times 3$ $\mathrm{mL}$ ), and dried under reduced pressure for $3 \mathrm{~h}$ to give 0.149 g (60\% based on $\mathrm{Pt})$. Anal. Calcd for $\mathrm{C}_{39} \mathrm{H}_{40} \mathrm{~N}_{2} \mathrm{FeP}_{2} \mathrm{Pt}_{2}$ : C 42.25, H 3.64, N 2.53\%. Found: C 42.56, H 3.57, N 2.34\%. IR $\left(\mathrm{cm}^{-1}\right), \mathrm{CH}_{2} \mathrm{Cl}_{2}: \mathrm{v}(\mathrm{CN}) 2136 \mathrm{~s} ; \mathrm{v}(\mathrm{CO}) 1976 \mathrm{~s} ; 1904 \mathrm{~m}$; and $1851 \mathrm{~m}$, br. ${ }^{1} \mathrm{H}-\mathrm{NMR}\left(\mathrm{CDCl}_{3}\right): \delta 1.30\left(\mathrm{~s}, 18 \mathrm{H}, \mathrm{CH}_{3}\right)$; $5.22\left[\mathrm{~m}, 2 \mathrm{H},{ }^{3} \mathrm{~J}(\mathrm{PtH}) 68 \mathrm{~Hz},{ }^{2} \mathrm{~J}(\mathrm{PH}) 11 \mathrm{~Hz}, \mathrm{CH}_{2}\right] ; 7.00-7.60$ (m, 20H, CH, Ph). ${ }^{13} \mathrm{C}-\left\{{ }^{1} \mathrm{H}\right\}$ NMR $\left(\mathrm{CD}_{2} \mathrm{Cl}_{2}\right): \delta 30.2(\mathrm{~s}$, $\left.\mathrm{CH}_{3}, \mathrm{Bu}^{\mathrm{t}}\right) ; 55.3\left[\mathrm{~m}, \mathrm{PCH}_{2},{ }^{1} J(\mathrm{PC}) 32 \mathrm{~Hz},{ }^{2} \mathrm{~J}(\mathrm{PtC}) 133 \mathrm{~Hz}\right]$; $57.4\left(\mathrm{~s}, C \mathrm{CH}_{3}\right) ; 128.4\left\{\mathrm{~m}, \mathrm{C}_{m}, \mathrm{Ph},\left[J(\mathrm{PC})+J\left(\mathrm{P}^{\prime} \mathrm{C}\right)\right] 10 \mathrm{~Hz}\right\}$; $130.1\left(\mathrm{~s}, \mathrm{C}_{p}, \mathrm{Ph}\right) ; 133.0\left\{\mathrm{~m}, \mathrm{C}_{o}, \mathrm{Ph},\left[J(\mathrm{PC})+J\left(\mathrm{P}^{\prime} \mathrm{C}\right)\right] 15\right.$ $\mathrm{Hz}\} ; 135.7\left\{\mathrm{~m}, \mathrm{C}_{i p s o}, \mathrm{Ph},\left[J(\mathrm{PC})+J\left(\mathrm{P}^{\prime} \mathrm{C}\right)\right] 50 \mathrm{~Hz}\right\} ; 144.1$ $\left[\mathrm{s}, C \mathrm{NBu}^{\mathrm{t}},{ }^{1} J(\mathrm{PtC}) 1380 \mathrm{~Hz}\right] ; 220.3(\mathrm{~s}, C \mathrm{O}) .{ }^{31} \mathrm{P}-\left\{{ }^{1} \mathrm{H}\right\}$ NMR: $\delta 4.18\left\{\mathrm{~s},{ }^{1} J(\mathrm{PtP}) 3671 \mathrm{~Hz},{ }^{2} J(\mathrm{PtP}) 75 \mathrm{~Hz},{ }^{2} J(\mathrm{PP})\right.$ $30 \mathrm{~Hz}, \mathrm{~N}^{\prime}=\left[{ }^{1} J(\mathrm{PtP})+{ }^{2} J(\mathrm{PtP})\right]=3596 \mathrm{~Hz} ;{ }^{1} J(\mathrm{PtPt})=850$ $\left.\mathrm{Hz}\} .{ }^{195} \mathrm{Pt}-\left\{{ }^{1} \mathrm{H}\right\}\left(\mathrm{CD}_{2} \mathrm{Cl}_{2}\right): \delta 457,{ }^{1} J(\mathrm{PtPt})=850 \mathrm{~Hz}\right]$.

A similar reaction was conducted under the same conditions as the preceding reaction by using $\left[\mathrm{Pt}_{3}(\mu-\right.$ $\left.\left.\mathrm{CNBu}^{\mathrm{t}}\right)_{3}\left(\mathrm{CNBu}^{\mathrm{t}}\right)_{3}\right] \quad(0.160 \mathrm{~g}, 0,148 \mathrm{mmol})$ and $\left[\mathrm{Fe}(\mathrm{CO})_{4}(\operatorname{dppm} P)\right](0.120 \mathrm{~g}, 0.217 \mathrm{mmol})$ in THF. This reaction gave $0.176 \mathrm{~g}(73 \%$ based on $\mathrm{Pt})$ of $\left[\mathrm{FePt}_{2}(\mu-\right.$ dppm $\left.)\left(\mathrm{CNBu}^{\mathrm{t}}\right)_{2}(\mathrm{CO})_{4}\right]$. IR and NMR data and analogous satisfactory elemental analyses, identical to those above, were obtained.

\section{Reaction of $\left[\mathrm{Pt}_{3}\left(\mu-\mathrm{CNBu}^{\mathrm{t}}\right)_{3}\left(\mathrm{CNBu}^{\mathrm{t}}\right)_{3}\right]$ with $\left[\mathrm{Fe}(\mathrm{CO})_{4}\right.$ $($ dppmP)] in petroleum ether/diethyl ether}

The $\left[\mathrm{Pt}_{3}\left(\mu-\mathrm{CNBu}^{\mathrm{t}}\right)_{3}\left(\mathrm{CNBu}^{\mathrm{t}}\right)_{3}\right]$ complex $(0.073 \mathrm{~g}, 0,068$ mmol) was slowly added to a stirred suspension of $\left[\mathrm{Fe}(\mathrm{CO})_{4}(\mathrm{dppm} P)\right](0.057 \mathrm{~g}, 0.103 \mathrm{mmol})$ in petroleum ether/diethyl ether $(1: 1,10 \mathrm{~mL})$ at $-5{ }^{\circ} \mathrm{C}$. The reaction mixture was stirred for 15 min until the formation of a black residue. The dark supernatant liquid was then removed, the residue washed several times with petroleum ether, and dried under vacuum, resulting in a dark green solid (0.031 $\mathrm{g}$ ). More product was present in the supernatant liquid, as shown by its NMR spectrum, but the yield has not been optimized. Anal. Calcd for $\mathrm{C}_{39} \mathrm{H}_{40} \mathrm{~N}_{2} \mathrm{FeP}_{2} \mathrm{Pt}_{2}$ : C 42.25, $\mathrm{H}$ 3.64, N 2.53\%. Found: C 42.00, H 3.65, N 2.53\%. IR $\left(\mathrm{cm}^{-1}\right)$, $\mathrm{CH}_{2} \mathrm{Cl}_{2}$ : v(CN) $2142 \mathrm{~s} ;$ br; v(CO) $2042 \mathrm{~s} ; 1980 \mathrm{~m}$, sh; 1970 s; $1945 \mathrm{~s} ; 1936 \mathrm{~s} ; 1840 \mathrm{~m}$; and $1812 \mathrm{~m}$, br. ${ }^{31} \mathrm{P}-\left\{{ }^{1} \mathrm{H}\right\} \mathrm{NMR}$ (THF): $\delta$-10.5 [s, PPt, ${ }^{1} J(\mathrm{PtP}) 3092 \mathrm{~Hz},{ }^{2} J(\mathrm{PtP}) 107 \mathrm{~Hz}$, $\left.{ }^{2} J(\mathrm{PP}) 48 \mathrm{~Hz}\right], 46.0\left[P F e, J\left(\mathrm{Pt}^{1} \mathrm{P}^{\mathrm{b}}\right)\right.$ and $J\left(\mathrm{Pt}^{2} \mathrm{P}^{\mathrm{b}}\right)$ not discernible, $\left.{ }^{2} J(\mathrm{PP}) 48 \mathrm{~Hz}\right]$ and the resonances assigned to $\left[\mathrm{FePt}_{2}(\mu-\right.$ dppm $)\left(\mathrm{CNBu}^{\mathrm{t}}\right)_{2}(\mathrm{CO})_{4}$ ] as the minor product.

\section{$X$-ray Data Collection, Structure Determination, and refinement for $\left[\mathrm{FePt}_{2}(\mu-d p p m)\left(\mathrm{CNBu}^{\mathrm{t}}\right)_{2}(\mathrm{CO})_{4}\right]$}

An orange crystal grown by slow diffusion of methanol into toluene, was mounted in an Enraf-Nonius CAD 4 diffractometer. The diffraction experiment was carried out at room temperature $(298 \mathrm{~K})$ using graphite monochromated $\operatorname{CuK} \alpha(\delta=1.5418 \AA)$ radiation. The cell constants

Table 2. Crystallographic Data for $\left[\mathrm{FePt}_{2}(\mu-\mathrm{dppm})\left(\mathrm{CNBu}^{\mathrm{t}}\right)_{2}(\mathrm{CO}) 4\right]$.

\begin{tabular}{|c|c|}
\hline formula & $\mathrm{C}_{39} \mathrm{H}_{40} \mathrm{~N}_{2} \mathrm{FeP}_{2} \mathrm{Pt}_{2}$ \\
\hline$M_{\mathrm{r}}$ & 1016.70 \\
\hline crystal size (mm) & $0.50 \times 0.25 \times 0.20$ \\
\hline system & monoclinic \\
\hline space group $(\mathrm{Z})$ & $P 2_{1} / \mathrm{n}, 4$ \\
\hline$a(\AA)$ & $11.031(1)$ \\
\hline$b(\AA)$ & $20.593(3)$ \\
\hline$c(\AA)$ & $18.129(2)$ \\
\hline$\beta(\operatorname{deg})$ & $91.32(1)$ \\
\hline$V\left(\AA^{3}\right)$ & $4117(1)$ \\
\hline$D_{\mathrm{c}}\left(\mathrm{mg} \mathrm{m}^{-3}\right)$ & 1.789 \\
\hline$\mu\left(\mathrm{Cu}-\mathrm{K}_{\alpha}\right)\left(\mathrm{mm}^{-1}\right)$ & 16.46 \\
\hline $2 \theta_{\max }(\operatorname{deg})$ & 59.88 \\
\hline hkl limits & $-12|\mathrm{~h}| 12,0|\mathrm{k}| 230|1| 14$ \\
\hline scan mode & $\omega / 2 \theta$ \\
\hline intensity variation $(\%)$ & 5.6 \\
\hline reflections collected & 6745 \\
\hline unique reflections & 5355 \\
\hline observed reflections, $I \geq 3 \sigma(\mathrm{I})$ & 4171 \\
\hline no. of parameters refined & 255, 277 ( 2 blocks) \\
\hline goodness-of-fit on F & 1.95 \\
\hline $\mathrm{R}_{\text {int }}$ & 0.0380 \\
\hline$R, R_{\mathrm{W}}$ & $0.0508,0.0558$ \\
\hline \multicolumn{2}{|l|}{$\mathrm{w}=1.00 /\left[\left(\mathrm{F}_{\mathrm{o}}\right)^{2}+0.00082 \mathrm{~F}_{\mathrm{o}}^{2}\right]$} \\
\hline absorption correction, $\mathrm{T}_{\operatorname{mim}}, \mathrm{T}_{\max }$ & $0.48,0.96$ \\
\hline$\Delta \rho_{\max }, \Delta \rho_{\operatorname{mim}}, \mathrm{e} \AA^{-3}$ & $1.60,-1.54$ \\
\hline extinction coefficient & 0.0006 \\
\hline
\end{tabular}


Table 3. Atomic Fractional Coordinates and $B_{\mathrm{eq}}\left(\AA^{2}\right)$ Values, with Esd's in parentheses, for $\left[\mathrm{FePt}_{2}(\mu-\mathrm{dppm})\left(\mathrm{CNBu}^{\mathrm{t}}\right)_{2}(\mathrm{CO})_{4}\right]$.

\begin{tabular}{lcccc}
\hline \multicolumn{1}{c}{ atom } & $\mathrm{x} / a$ & $\mathrm{y} / b$ & $\mathrm{z} / c$ & $B_{\mathrm{eq}} a$ \\
\hline $\mathrm{Pt}(1)$ & $0.0536(0)$ & $0.1636(0)$ & $0.2705(0)$ & $2.61(2)$ \\
$\mathrm{Pt}(2)$ & $0.0870(0)$ & $0.1666(0)$ & $0.1304(0)$ & $2.64(2)$ \\
$\mathrm{Fe}$ & $0.0019(2)$ & $0.2662(1)$ & $0.1966(1)$ & $3.10(6)$ \\
$\mathrm{P}(1)$ & $0.0745(2)$ & $0.0562(1)$ & $0.2823(1)$ & $2.39(8)$ \\
$\mathrm{P}(2)$ & $0.1723(3)$ & $0.0682(1)$ & $0.1343(1)$ & $2.73(8)$ \\
$\mathrm{O}(\mathrm{A} 1)$ & $-0.054(1)$ & $0.3375(4)$ & $0.0614(6)$ & $6.0(4)$ \\
$\mathrm{O}(\mathrm{A} 2)$ & $-0.068(1)$ & $0.3540(5)$ & $0.3152(7)$ & $9.0(6)$ \\
$\mathrm{O}(\mathrm{A} 3)$ & $-0.2106(8)$ & $0.1809(5)$ & $0.1918(6)$ & $6.1(4)$ \\
$\mathrm{O}(\mathrm{A} 4)$ & $0.2659(9)$ & $0.2799(5)$ & $0.2150(6)$ & $6.8(4)$ \\
$\mathrm{N}(1)$ & $0.033(1)$ & $0.2099(5)$ & $0.4326(6)$ & $4.7(4)$ \\
$\mathrm{N}(2)$ & $0.089(1)$ & $0.2068(6)$ & $-0.0343(6)$ & $5.0(4)$ \\
$\mathrm{C}$ & $0.0889(9)$ & $0.0144(40$ & $0.1928(5)$ & $2.8(3)$ \\
$\mathrm{C}(\mathrm{A} 1)$ & $-0.031(1)$ & $0.3106(5)$ & $0.1159(7)$ & $3.6(4)$ \\
$\mathrm{C}(\mathrm{A} 2)$ & $-0.036(1)$ & $0.3202(5)$ & $0.2652(8)$ & $5.1(5)$ \\
$\mathrm{C}(\mathrm{A} 3)$ & $-0.120(1)$ & $0.2105(6)$ & $0.1958(7)$ & $4.4(5)$ \\
$\mathrm{C}(\mathrm{A} 4)$ & $0.162(1)$ & $0.2719(5)$ & $0.2055(7)$ & $4.4(5)$ \\
$\mathrm{C}(1)$ & $0.0381(9)$ & $0.1885(6)$ & $0.3737(6)$ & $3.3(4)$ \\
$\mathrm{C}(2)$ & $0.0913(9)$ & $0.1875(6)$ & $0.0272(6)$ & $3.8(4)$ \\
$\mathrm{C}(3)$ & $0.035(2)$ & $0.2369(9)$ & $0.5061(8)$ & $6.6(7)$ \\
$\mathrm{C}(4)$ & $0.071(2)$ & $0.2339(9)$ & $-0.1087(8)$ & $7.2(7)$ \\
$\mathrm{C}(5)$ & $-0.055(2)$ & $0.257(1)$ & $-0.119(1)$ & $10 .(1)$ \\
$\mathrm{C}(6)$ & $0.156(2)$ & $0.290(1)$ & $-0.116(1)$ & $15 .(1)$ \\
$\mathrm{C}(7)$ & $0.112(3)$ & $0.184(1)$ & $-0.163(1)$ & $17 .(1)$ \\
$\mathrm{C}(8)$ & $-0.020(4)$ & $0.298(1)$ & $0.506(1)$ & $26 .(1)$ \\
$\mathrm{C}(9)$ & $0.151(2)$ & $0.235(2)$ & $0.534(10$ & $22 .(1)$ \\
\hline & & & &
\end{tabular}

were determinated from a list of 25 selected reflections by using automatic search, indexing factors, and least-squares routines. The data were corrected for Lorentz and polarization factors, and a semiempirical absorption correction was applied $^{19}$. The coordinates of the iron and platinum atoms were obtained by the Patterson method. An alternating sequence of least-squares refinement and difference Fourier maps revealed the positions of all remaining atoms. The hydrogen atoms were introduced in calculated positions $\left(\mathrm{d}_{\mathrm{C}-\mathrm{H}}=1.08 \AA\right.$ ) and the coordinates were recalculated after each refinement cycle with fixed isotropic parameters $\left(B=6.0 \AA^{2}\right)$. Final refinement was carried out using anisotropic displacement parameters for all non-hydrogen atoms. All calculations were performed using the SHELX-76 ${ }^{20}$ and SHELXS ${ }^{21}$ packages of programs. Atomic scattering factors were taken from the usual source ${ }^{22}$. A summary of the data collection is given in Table 2 .

\begin{tabular}{lcccc}
\hline atom & $\mathrm{x} / a$ & $\mathrm{y} / b$ & $\mathrm{z} / c$ & $B_{\mathrm{eq}} a$ \\
\hline $\mathrm{C}(10)$ & $-0.040(3)$ & $0.195(2)$ & $0.556(10$ & $18 .(1)$ \\
$\mathrm{C}(1 \mathrm{~A})$ & $0.325(1)$ & $0.0675(5)$ & $0.1740(5)$ & $3.3(3)$ \\
$\mathrm{C}(2 \mathrm{~A})$ & $0.385(1)$ & $0.1255(5)$ & $0.1860(6)$ & $3.3(4)$ \\
$\mathrm{C}(3 \mathrm{~A})$ & $0.501(1)$ & $0.1274(7)$ & $0.2140(7)$ & $4.7(5)$ \\
$\mathrm{C}(4 \mathrm{~A})$ & $0.562(1)$ & $0.0704(8)$ & $0.2344(8)$ & $5.5(6)$ \\
$\mathrm{C}(5 \mathrm{~A})$ & $0.501(1)$ & $0.0121(7)$ & $0.2225(7)$ & $4.9(5)$ \\
$\mathrm{C}(6 \mathrm{~A})$ & $0.386(1)$ & $0.0108(5)$ & $0.1918(6)$ & $3.8(4)$ \\
$\mathrm{C}(7 \mathrm{~A})$ & $0.193(1)$ & $0.0221(50$ & $0.0492(6)$ & $3.2(4)$ \\
$\mathrm{C}(8 \mathrm{~A})$ & $0.136(1)$ & $-0.0351(6)$ & $0.0327(6)$ & $4.4(4)$ \\
$\mathrm{C}(9 \mathrm{~A})$ & $0.157(1)$ & $-0.0673(6)$ & $-0.0330(7)$ & $5.0(5)$ \\
$\mathrm{C}(10 \mathrm{~A})$ & $0.232(1)$ & $-0.0425(7)$ & $-0.0821(8)$ & $6.2(6)$ \\
$\mathrm{C}(11 \mathrm{~A})$ & $0.285(2)$ & $0.0170(8)$ & $-0.0696(9)$ & $9.1(8)$ \\
$\mathrm{C}(12 \mathrm{~A})$ & $0.267(2)$ & $0.0478(7)$ & $-0.0024(8)$ & $7.7(7)$ \\
$\mathrm{C}(1 \mathrm{~B})$ & $0.2012(9)$ & $0.0243(4)$ & $0.3362(5)$ & $2.5(3)$ \\
$\mathrm{C}(2 \mathrm{~B})$ & $0.215(1)$ & $-0.0419(5)$ & $0.3480(6)$ & $3.3(4)$ \\
$\mathrm{C}(3 \mathrm{~B})$ & $0.313(1)$ & $-0.0664(7)$ & $0.3874(7)$ & $5.0(5)$ \\
$\mathrm{C}(4 \mathrm{~B})$ & $0.398(1)$ & $-0.0217(6)$ & $0.4147(7)$ & $4.5(5)$ \\
$\mathrm{C}(5 \mathrm{~B})$ & $0.3986(1)$ & $0.0435(5)$ & $0.4059(7)$ & $4.1(4)$ \\
$\mathrm{C}(6 \mathrm{~B})$ & $0.286(1)$ & $0.0662(5)$ & $0.3657(6)$ & $3.4(4)$ \\
$\mathrm{C}(7 \mathrm{~B})$ & $-0.054(1)$ & $0.0157(5)$ & $0.3262(6)$ & $3.0(4)$ \\
$\mathrm{C}(8 \mathrm{~B})$ & $-0.076(1)$ & $0.0316(8)$ & $0.3978(8)$ & $7.0(6)$ \\
$\mathrm{C}(9 \mathrm{~B})$ & $-0.167(2)$ & $0.0025(8)$ & $0.4368(9)$ & $7.9(7)$ \\
$\mathrm{C}(10 \mathrm{~B})$ & $-0.238(1)$ & $-0.0420(8)$ & $0.402(1)$ & $6.8(7)$ \\
$\mathrm{C}(11 \mathrm{~B})$ & $-0.221(1)$ & $-0.0588(7)$ & $0.3324(9)$ & $5.6(6)$ \\
$\mathrm{C}(12 \mathrm{~B})$ & $-0.125(1)$ & $-0.0295(6)$ & $0.2934(7)$ & $4.4(5)$ \\
\hline & & & &
\end{tabular}

${ }^{a} B_{\mathrm{eq}}=8 / 3 \pi^{2} \Sigma_{i} \Sigma_{j} U_{i j} \mathrm{a}_{i} * \mathrm{a}_{j} * \mathrm{a}_{i} \mathbf{a}_{j}$.

\section{Acknowledgment}

We are very grateful to the $\mathrm{CNPq} / \mathrm{PADCT}$ for financial support. We Thank Dr. Brian Mann for several suggestions and for recording some of the NMR spectra.

Supporting Information Available: Tables of hydrogen atomic fractional coordinates, interatomic distances, anisotropic thermal parameters. Ordering information is given on any current masthead page.

\section{References}

1. a) Stone, F.G.A. Acc. Chem. Res. 1981, 14, 318; Inorg. Chim Acta 1981, 50, 33; Angew. Chem. Int. Ed. Engl. 1984, 23, 89. b) Farrugia, L.J.; Howard, J.A.; Mitrprachachon, K.P.; Stone, F.G.A.; Woodward, P. J. Chem. Soc., Dalton Trans. 1981, 1134. c) Adams, R.D.; Arafa, I.; Chen, G.; Lii, J.-C.; Wang, J.-G.; Organometallics 1990, 9, 2350. d) Braustein, P. Mat. 
Chem. Phys. 1991, 29, 33. e) Farrugia, L.J. Adv. Organomet. Chem. 1990, 31, 301. f) Rashidi, M.; Vittal, J. J.; Puddephatt, R.J. J. Chem. Soc. Dalton Trans. 1994, 1283.

2. a) Chatt, J.; Chini, P. J. Chem. Soc. A, 1970, 538. b) Bender, R.; Braustein, P. J. Chem. Soc., Chem. Commun. 1983, 334.

3. a) Puddephatt, R.J.; Manojlovic-Muir, L.; Muir, K.W.; Polyhedron 1990, 9, 2767. b) Puddephatt, R.J. Chem. Soc. Rev. 1983, 12, 99. c) Chaudret, B.; Delavaux, B.; Poilblanc, R. Coord. Chem. Revs, 1988, 86, 191.

4. a) Braustein, P.; Bellefon, C.M.; Ries, M. J. Organomet. Chem. 1984, 262, C14. b) Braustein, P.; Jud, J.-M.; Dusausoy, Y.; Fisher, J. Organometallics 1983, 2,180 .

5. a) Grossel, M.C.; Moulding, R.P.; Seddon, K.R. J. Organomet. Chem. 1983, 253, C50. b) Braustein, P.; Guarino, N.; Bellefon, C.M.; Richert; J-L. Angew. Chem. Int. Ed. Engl. 1987, 26, 88. c) Braustein, P.; Richert, J.-L.; Dusausoy, Y. J. Chem. Soc., Dalton Trans. 19903801.

6. Fontaine, X.L.R; Jacobsen, G.B.; Shaw, B.L.; Thornton-Pett, M. J. Chem. Soc., Dalton Trans. 1988, 741.

7. Brown, M.P.; Puddephatt, R.J.; Rashidi, M. J. Chem. Soc., Dalton Trans. 1977, 951.

8. a) Tulip, T.H.; Yamagata, T.; Yoshida, T.; Wilson, R.D.; Ibers, J.A.; Otsuka, S. Inorg. Chem. 1979, 18, 2239. b) Bender, R.; Braustein, P.; Jud, J-M.; Dusausoy, Y. Inorg. Chem. 1984, 23, 4489; and references cited therein.

9. Brown, M.P.; Fisher, J.R.; Franklin, S.J.; Puddephatt, R.J.; Seddon, K.R. J. Organomet. Chem. 1978, 161, C46.
10.Johnson, C.K. ORTEP; Report ORNL-3794; Oak Ridge National Laboratory: Oak Ridge, TN, 1965.

11. Crabtree, R.H.; Lavin, M. Inorg. Chem. 1986, $25,805$.

12. Puddephatt, R.J.; Rashidi, M.; Vittal, J.J. J. Chem. Soc., Dalton Trans. 1991, 2875; and references cited therein.

13. a) Churchill, M.R.; DeBoer, B.G.; Rotella, F.J. Inorg. Chem. 1976, 15, 1843. b) Cotton, F.A.; Troup, J.M. J. Am. Chem. Soc. 1974, 96, 4155. c) Albano, V.G.; Ciani, G. J. Organomet. Chem. 1974, 66, 311. d) Briant, C.E.; Gilmour, D.I.; Mingos, D.M.; Warlde, R.W.M. J. Chem. Soc., Dalton Trans. 1985, 1693.

14. Manojlovic-Muir, L.; Mirza, H.A.; Sadiq, N.; Puddephatt, R.J. Inorg. Chem. 1993, 32, 117. Olmstead, M.M.; Lee, C-L.; Balch, A.L. Inorg. Chem. 1982, 21, 2712.

15. Morton, D.A.V.; Orpen, A.G. J. Chem. Soc., Dalton Trans. 1992, 641.

16. Green, M.; Howard, J.A.K.; Spencer, J.L.; Stone, F.G.A. J. Chem. Soc., Dalton Trans. 1977, 271.

17. Green, M.; Howard, J.A.K.; Murray, M.; Spencer, J.L.; Stone, F.G.A. J. Chem. Soc., Dalton Trans. 1977, 1509.

18. Jacobsen, G.B.; Shaw, B.L.; Thornton-Pett, M. J. Chem. Soc., Dalton Trans. 1987, 1509.

19. Walker, N.; Stuart, D. Acta Cryst. 1983, A39, 158.

20. Sheldrick, G.M.; SHELX-76, Program for crystal structure determination; University of Cambridge, England, 1976.

21. Sheldrick, G.M.; SHELXS-86, Program for the solution of crystal structure; Universität Göttingen, Germany, 1985.

22. International Tables for X-ray Crystallography; Kynoch Press; Birmingham, UK, 1974, Vol 4.

FAPESP helped in meeting the publication costs of this article 